تأثير تذذية الاعسوقة ذات التسع نقاط Coccinella novemnotata Herbst والدعسوقة ذات الاحد

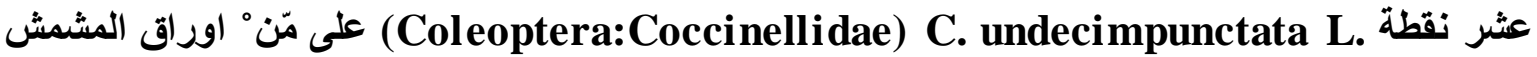
(Hemiptera: Aphididae) Hyalopterus pruni Geoffr.

$$
\begin{aligned}
& \text { للمفترسين }
\end{aligned}
$$

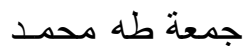

$$
\begin{aligned}
& \text { قسم وقاية النبات ـ كلية الزر اعة والغابات ـ جامعة الموصل ـ الموصل ـ العراق } \\
& \text { Email:jumaa_taha@yahoo.com }
\end{aligned}
$$

\title{
الخلاصة إنمة
}

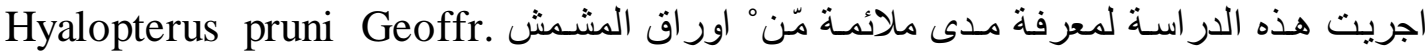

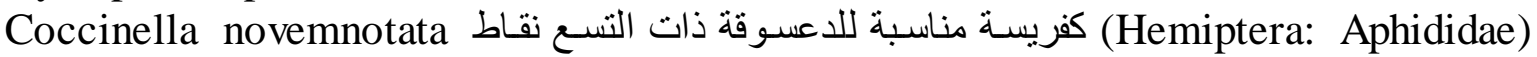

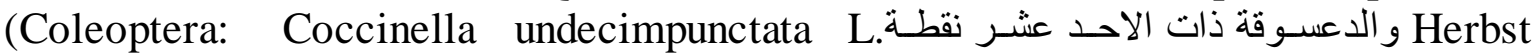
(Coccinellidae)

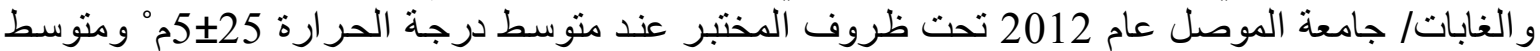

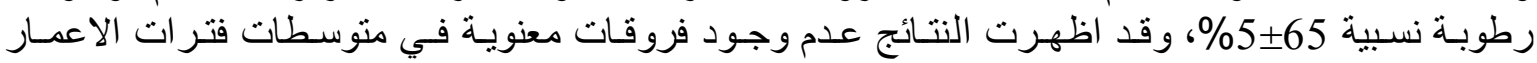

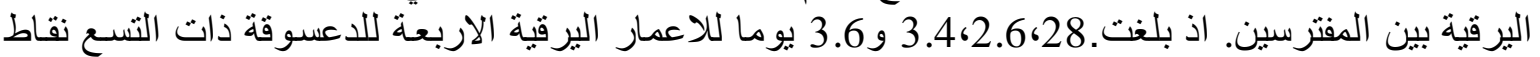

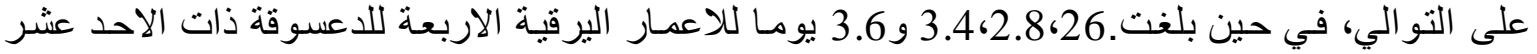

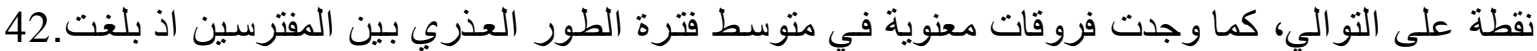

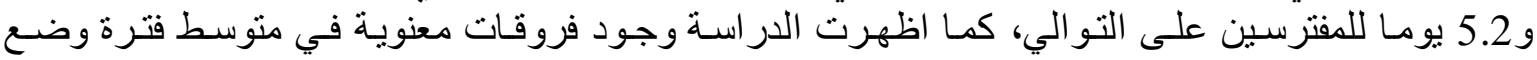

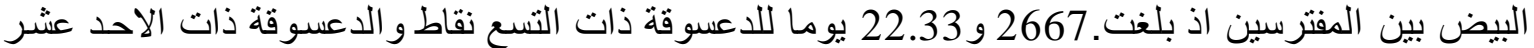

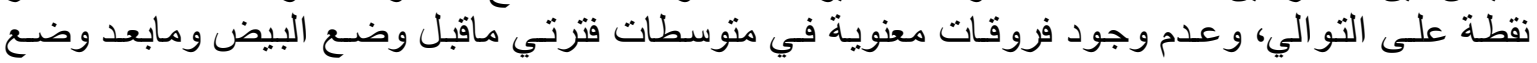

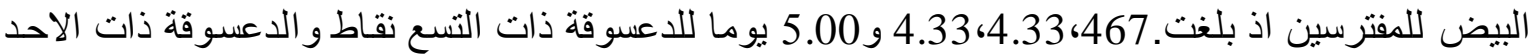

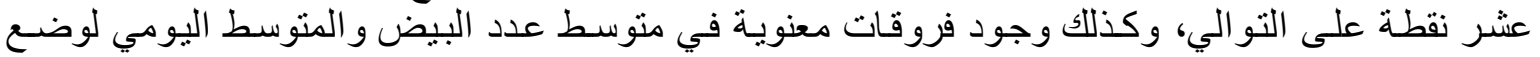

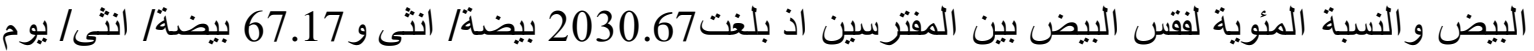

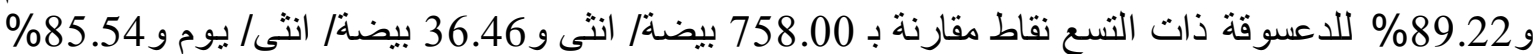

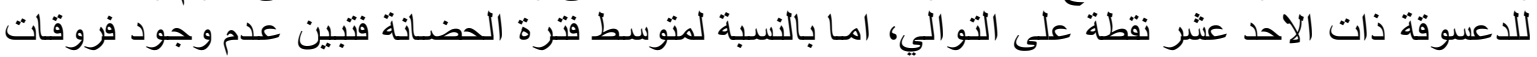

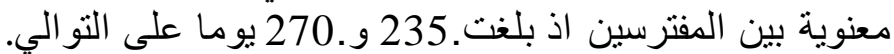
كلمات داله: الدعسوقة ذات التسع نقاط، الدعسوقة ذات الاحد عشر نقطة، مّنْ اوراق المشمش، الصفات الحياتية

\section{(المقدمة}

تاريخ تسلم البحث:2014/1/8، وقبوله 2017/12/17

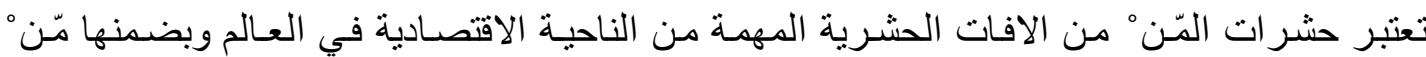

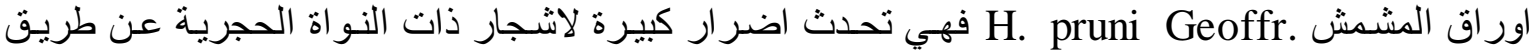

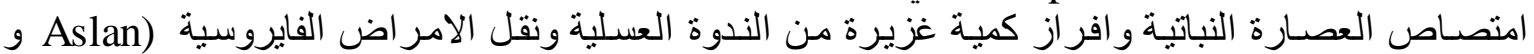

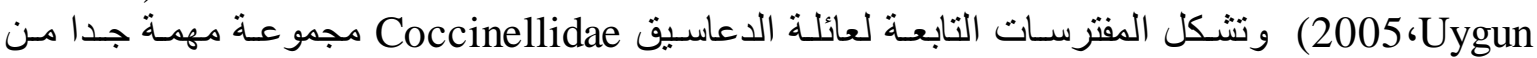

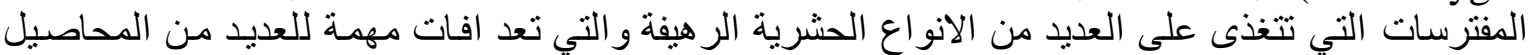

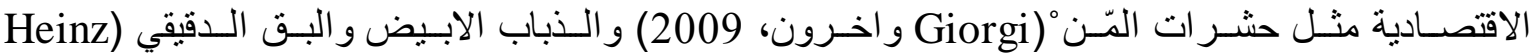

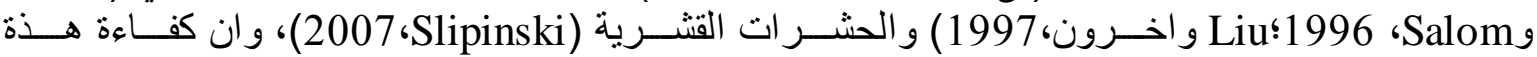

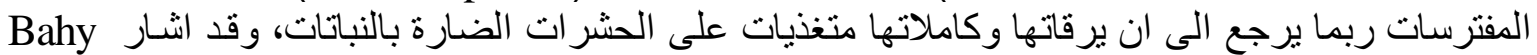
El-Din

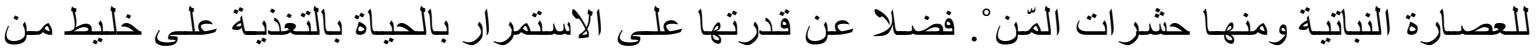

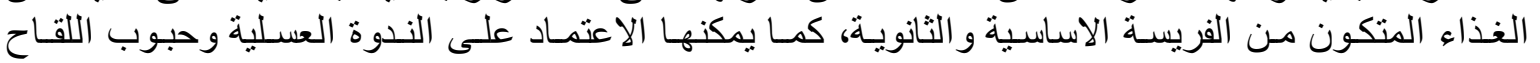

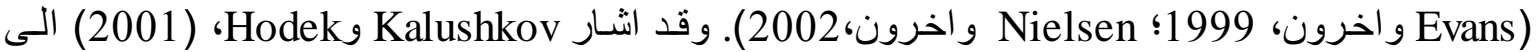

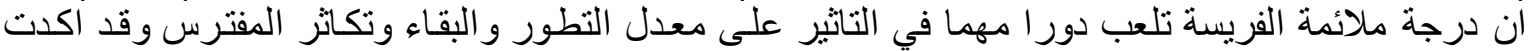
النتائج الني توصل اليها كل من Mari واخرون، (2005) و Solangi و اخرون، (2007) وRoy و واخرون، 
(2010) هذة الحقيقة حيث وجدو ا ان نوع الفريسة بلعب دور ا مهما في معدل التكاثر ونضـج البيض وتطور

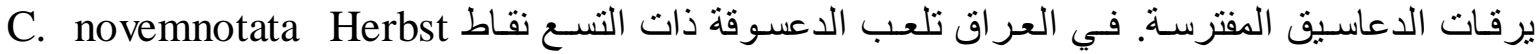

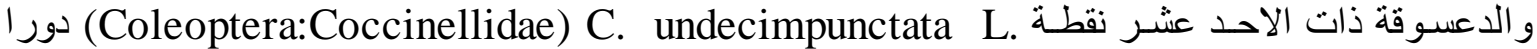

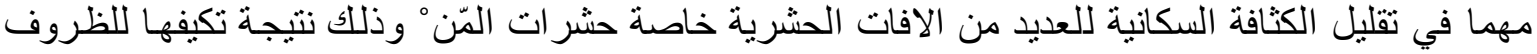

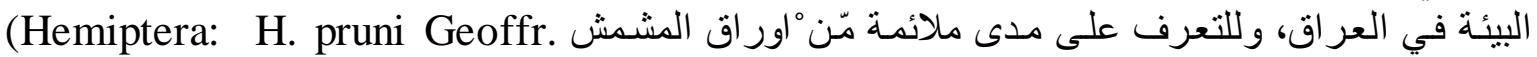

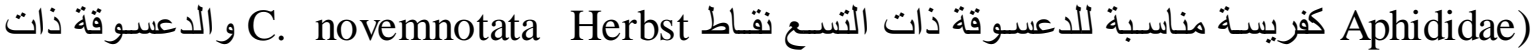

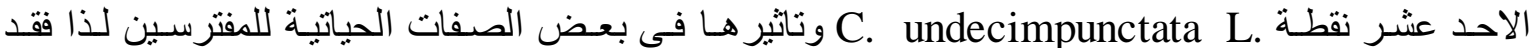

\section{مواد البحث وطر ائقه}

اجريت هذة الدراسة.

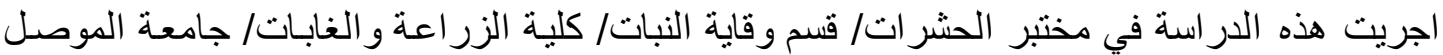

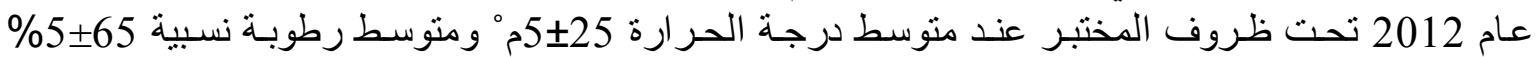

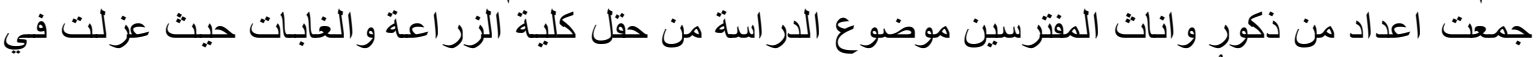

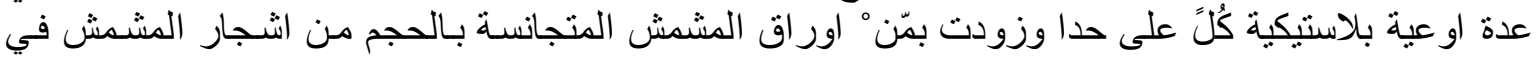

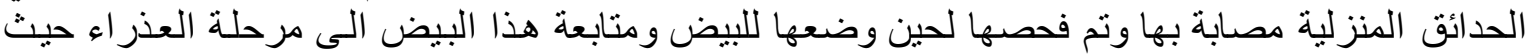

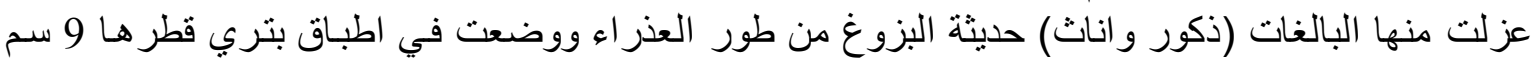

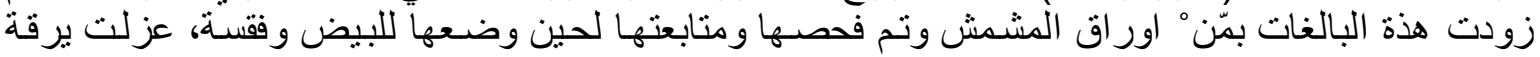

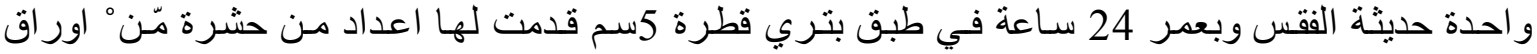

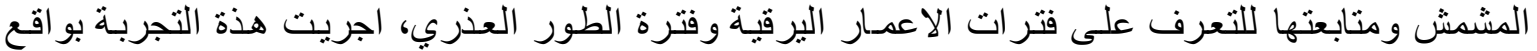

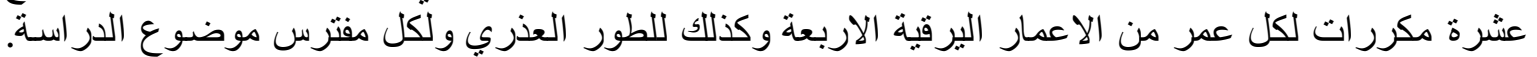

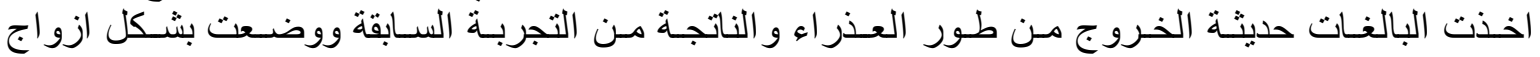

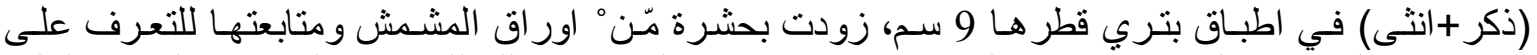

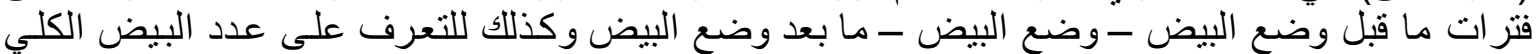

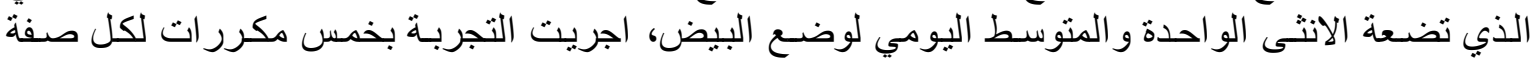
ولكل مفترس. عزلت البيوض التي وضعتها الاناث خلال فترة وضع البيض البض في كل مكرر يوميا وتم فحصهـا

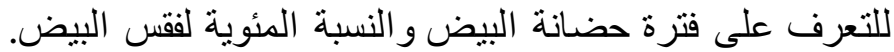

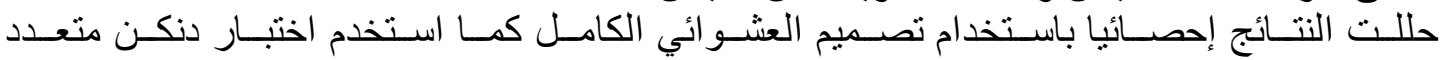

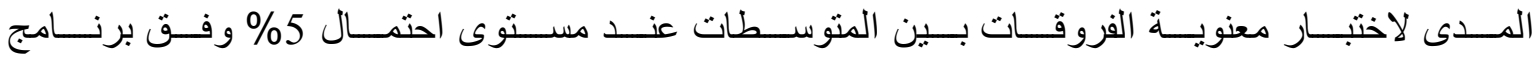

\section{النتائج و المناقشة}

.(1993،Anonymous) SAS

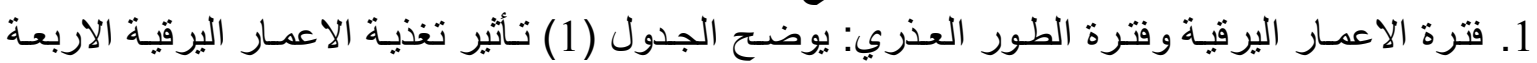

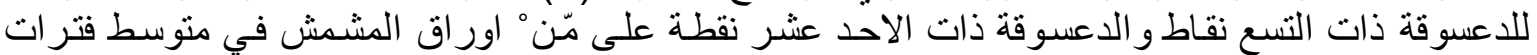

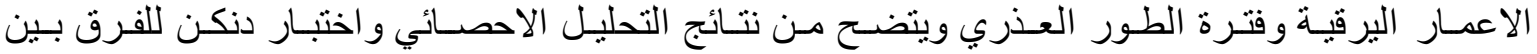

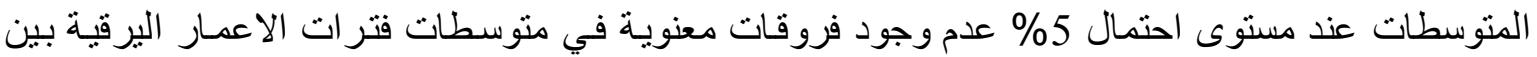

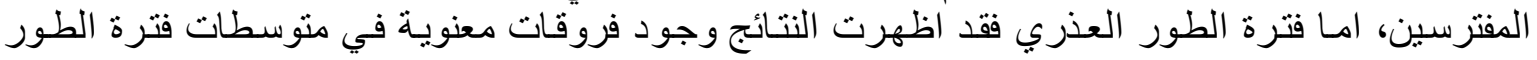

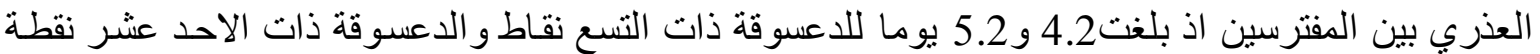

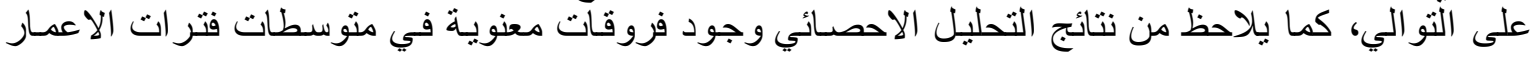

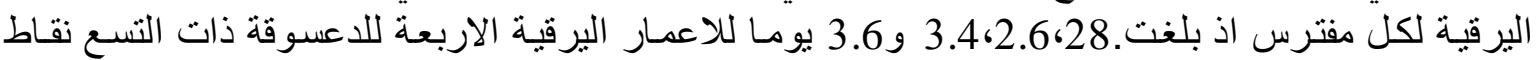

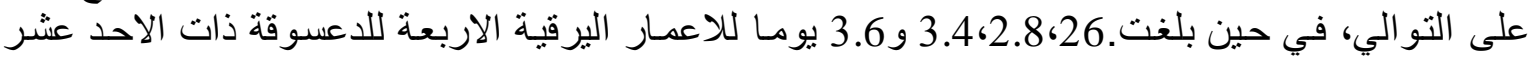

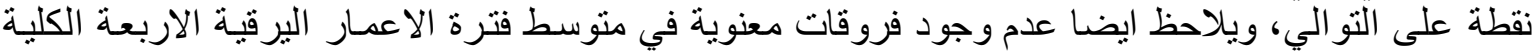

للمفترسين.

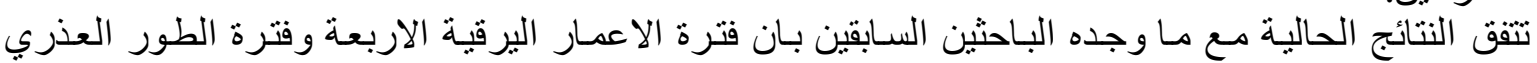

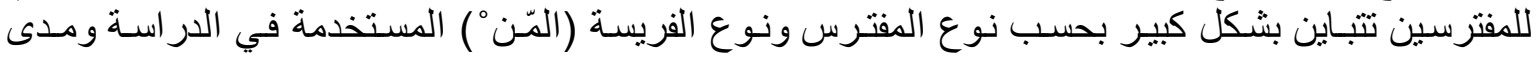

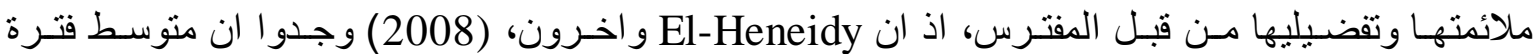

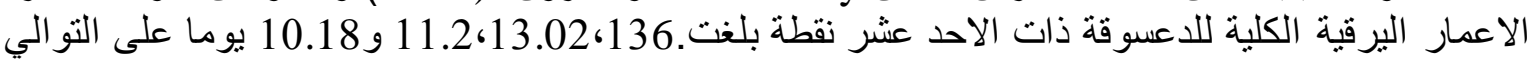

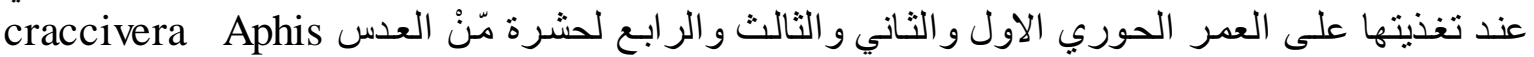
Adonia variegate Goeze.Koch 


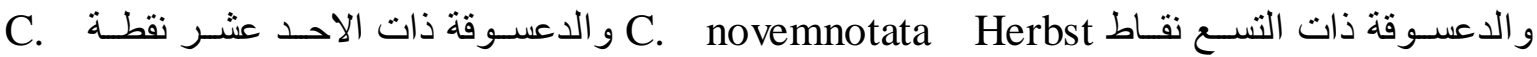
undecimpunctata L.

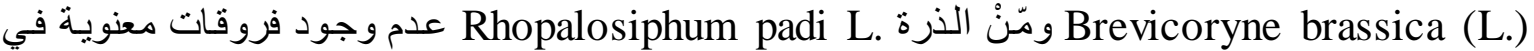

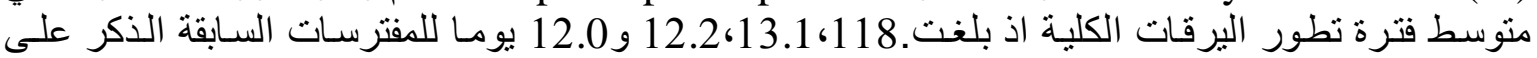

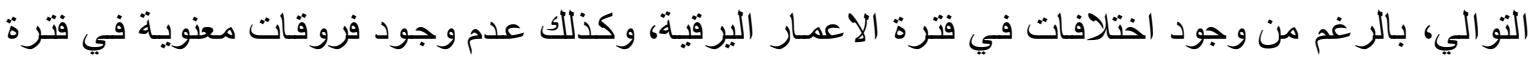
الطور العذري للمفترسات العلاه.

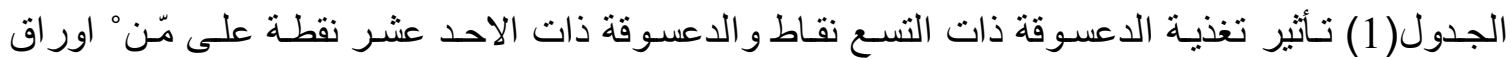

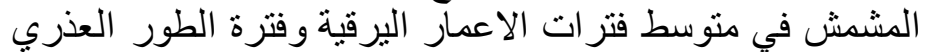

Table (1) Feeding effects of the C. novemnotata and C. undecimpunctata on the H. pruni Geoffr. on mean of larval and pupal stage period

\begin{tabular}{|c|c|c|c|c|}
\hline \multicolumn{2}{|c|}{ الدعسوقة ذات الاحد عشر نقطة } & \multicolumn{2}{|c|}{$\begin{array}{c}\text { الدعسوقة ذات التسع نقاط } \\
\text { C. novemnotata } \\
\end{array}$} & \multirow{2}{*}{$\begin{array}{c}\text { فترة الاعمار اليرقية / بوم / } \\
\text { Larval stage } \\
\text { period/day }\end{array}$} \\
\hline $\begin{array}{l}\text { SDEל المتوسط } \\
\text { Mean+S.D }\end{array}$ & المدى Rang & $\begin{array}{l}\text { SD士 المتوسط } \\
\text { Mean+S.D }\end{array}$ & Rang المدى & \\
\hline$* 2.60 \pm 0.55 \mathrm{c}$ & $2-3$ & $2.80 \pm 0.45 b c$ & $2-3$ & $\begin{array}{c}\text { st. العمر اليرقي الاول } \\
\text { 1stage } \\
\end{array}$ \\
\hline $2.80 \pm 0.45 b c$ & $2-3$ & $2.60 \pm 0.55 \mathrm{c}$ & $2-3$ & $\begin{array}{l}\text { nd. العمر اليرقي الثاني } \\
\text { 2stage }\end{array}$ \\
\hline $3.40 \pm 0.55 \mathrm{ab}$ & $3-4$ & $3.40 \pm 0.55 \mathrm{ab}$ & $3-4$ & $\begin{array}{c}\text { rd. العمر اليرقي الثالث } \\
\text { 3stage }\end{array}$ \\
\hline $3.60 \pm 0.55 a$ & $3-4$ & $3.60 \pm 0.55 a$ & $3-4$ & $\begin{array}{l}\text { th. العمر اليرقي الرابع } \\
\text { 4stage }\end{array}$ \\
\hline \multicolumn{2}{|c|}{$12.40 \mathrm{a}$} & \multicolumn{2}{|c|}{$12.40 \mathrm{a}$} & $\begin{array}{l}\text { المتو سط العام } \\
\text { Total mean } \\
\end{array}$ \\
\hline $5.20 \pm 0.84 a$ & $5-6$ & $4.20 \pm 0.45 b$ & $3-5$ & $\begin{array}{c}\text { العذري/ يوم الطور pupal } \\
\text { stage period/day }\end{array}$ \\
\hline
\end{tabular}

*Means followed by different letters are significantly different at $\mathrm{P}=0.05$.

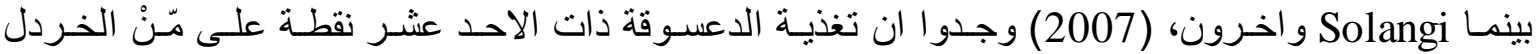
Lipaphis erysimi Kalt.

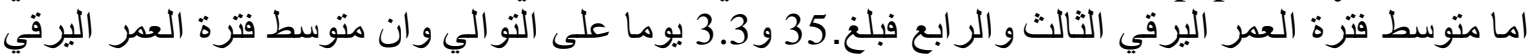

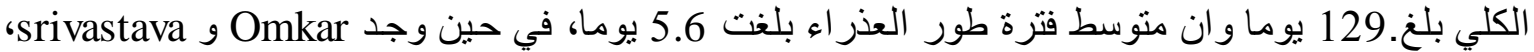

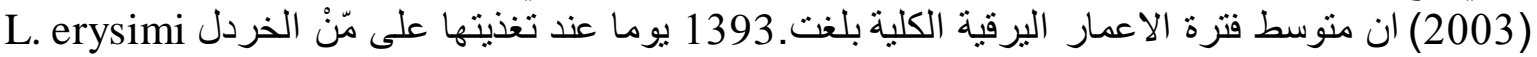

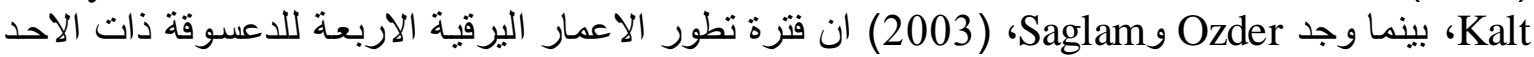

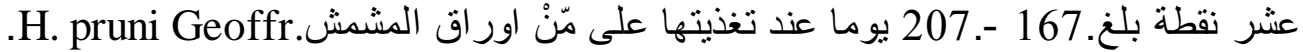

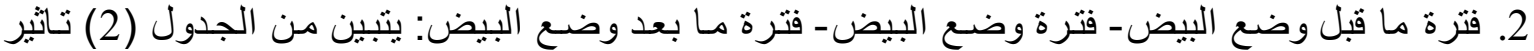

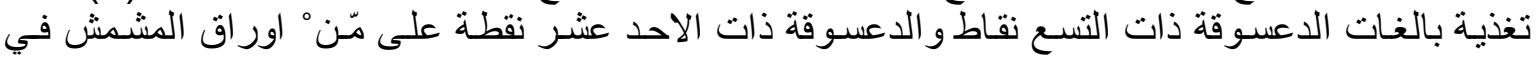

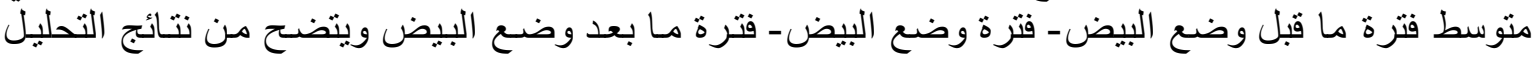

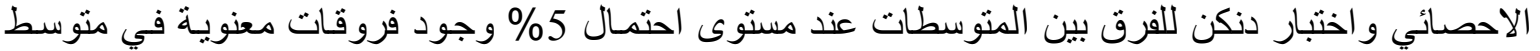

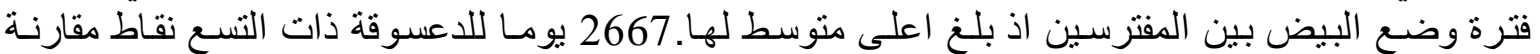

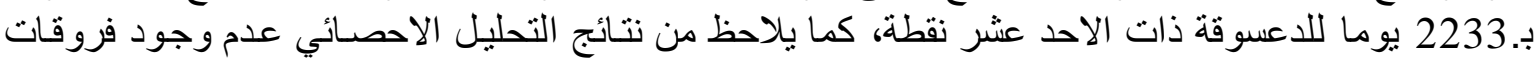

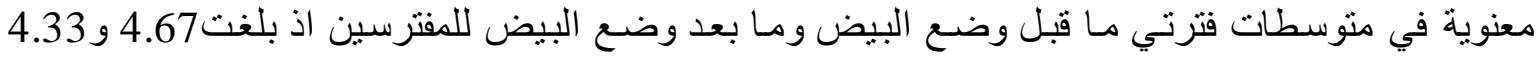


يوما للدعسوقة ذات التسع نقاط على التو الي، بينما بلغت.433 و 5.00 يو ما للاعسوقة ذات الاحد عشر نقطة على التو الي.

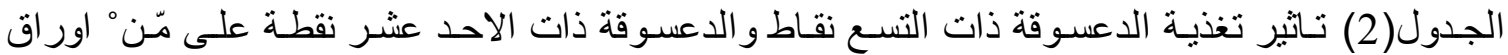

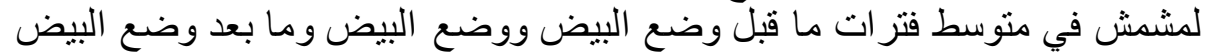

Table (2) Feeding effects of the C. novemnotata and C. undecimpunctata on the H. pruni Geoffr. on means of Pre-oviposition - Oviposition and Postoviposition period

\begin{tabular}{|c|c|c|c|c|}
\hline \multicolumn{2}{|c|}{ الدعسوقة ذات الاحد عشر نقطة } & \multicolumn{2}{|c|}{ الدعسوقة ذات التسع نقاط } & \multirow[t]{2}{*}{$\begin{array}{l}\text { نوع المفترس } \\
\text { Kind of predator }\end{array}$} \\
\hline $\begin{array}{l}\text { SD士المتوسط } \\
\text { Mean }+ \text { S.D }\end{array}$ & المدى Rang & 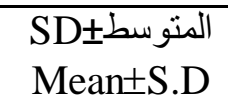 & Rang المدى & \\
\hline *4.33 $\pm 0.58 \mathrm{c}$ & $4-5$ & $4.67 \pm 0.58 \mathrm{c}$ & $4-5$ & $\begin{array}{c}\text { فترة ما قبل وضيض يوم } \\
\text { Pre-oviposition } \\
\text { period/day }\end{array}$ \\
\hline $22.33 \pm 2.89 b$ & $19-24$ & $26.67 \pm 3.06 \mathrm{a}$ & $24-30$ & فترة وضع البيض/ يوم \\
\hline $5.00 \pm 1.00 \mathrm{c}$ & $4-6$ & $4.33 \pm 0.58 \mathrm{c}$ & $4-5$ & $\begin{array}{c}\text { فترة ما بعد وضي يوم } \\
\text { وضيض } \\
\text { Post- oviposition } \\
\text { period/day }\end{array}$ \\
\hline
\end{tabular}

*Means followed by different letters are significantly different at $\mathrm{P}=0.05$.

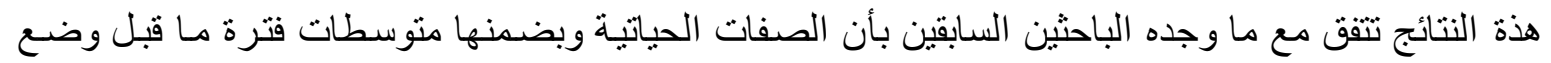

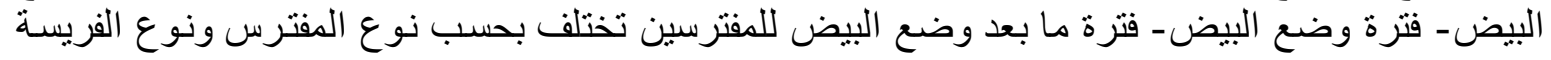

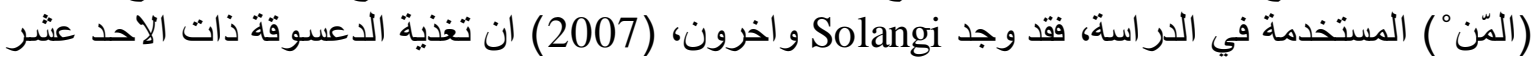

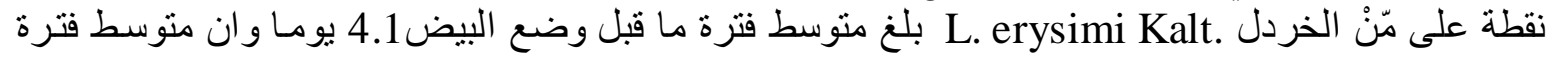

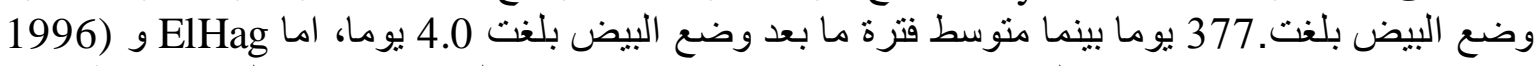

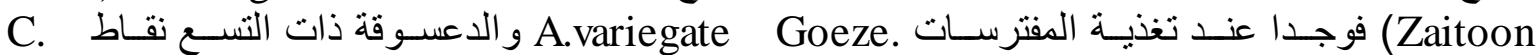
novemnotata Herbst

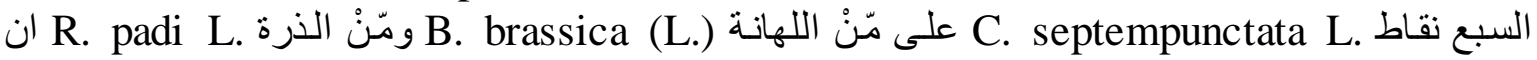

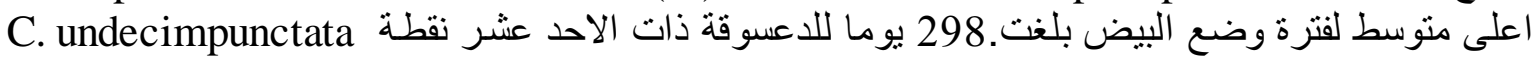

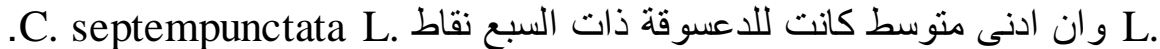

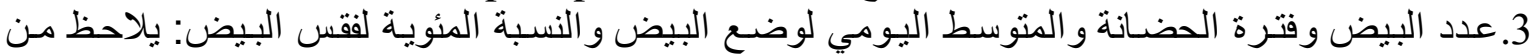

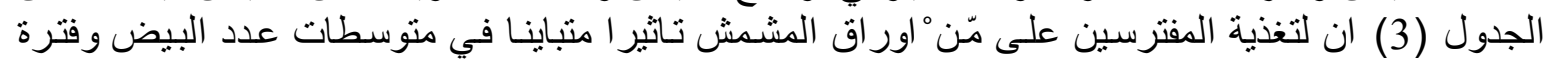

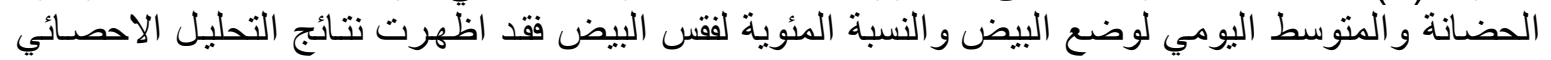

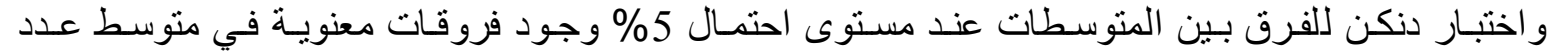

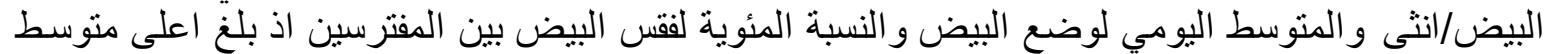

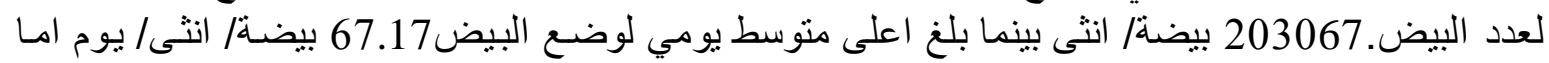

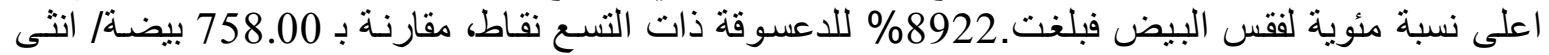

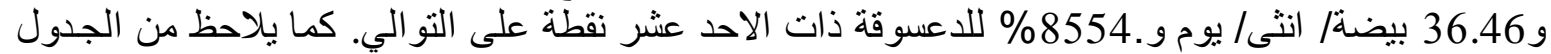

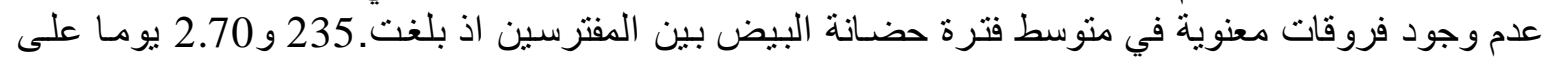
التو البي. 


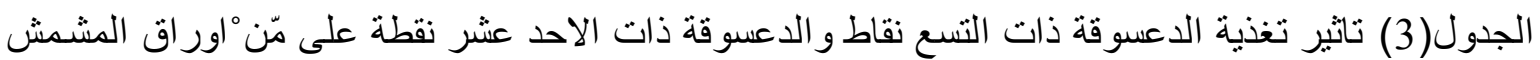

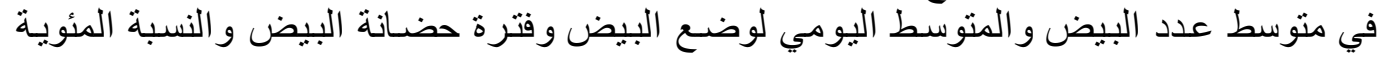

$$
\text { لفقس البيض متص فيط }
$$

Table (3) Feeding effects of the C. novemnotata and C. undecimpunctata on the $\mathrm{H}$. pruni Geoffr. on mean number of egg/female, daily deposited of egg, incubation period and hatching percentage

\begin{tabular}{|c|c|c|c|c|}
\hline \multicolumn{2}{|c|}{ الدعسوقة ذات الاحد عشر نقطة } & \multicolumn{2}{|c|}{$\begin{array}{c}\text { الدعسوقة ذات التسع نقاط } \\
\text { C. novemnotata }\end{array}$} & \multirow{2}{*}{ الصفات المدروسة } \\
\hline 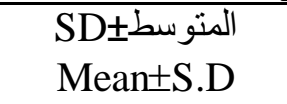 & المدى Rang & $\begin{array}{l}\text { SD } \pm \text { المنوسط } \\
\text { Mean } \pm \text { S.D }\end{array}$ & المدى Rang & \\
\hline${ }^{*} 758.00 \pm 32.19 b$ & 728-792 & $2030.67 \pm 342.25 a$ & $963-2419$ & $\begin{array}{l}\text { عدد البيض/انثى } \\
\text { Number of } \\
\text { egg/female }\end{array}$ \\
\hline $36.46 \pm 17.95 b$ & $4-72$ & $67.17 \pm 39.14 \mathrm{a}$ & $10-161$ & $\begin{array}{c}\text { لوضع البيض اليومي } \\
\text { Daily } \\
\text { deposited of } \\
\text { egg/female/day }\end{array}$ \\
\hline $2.70 \pm 0.73 \mathrm{a}$ & $2-3$ & $2.35 \pm 0.54 a$ & $2-3$ & فترة حضانة البيض/ \\
\hline $85.54 \pm 3.90 \mathrm{~b}$ & $80-94$ & $89.22 \pm 5.13 \mathrm{a}$ & $80-100$ & $\begin{array}{c}\text { النسبة المئوية لفقبض\% } \\
\text { Hatching } \\
\text { percent. \% }\end{array}$ \\
\hline
\end{tabular}

*Means followed by different letters in each row are significantly different at $\mathrm{P}=0.05$.

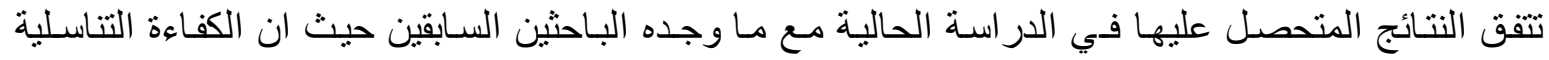

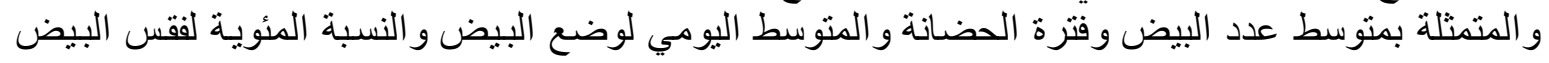

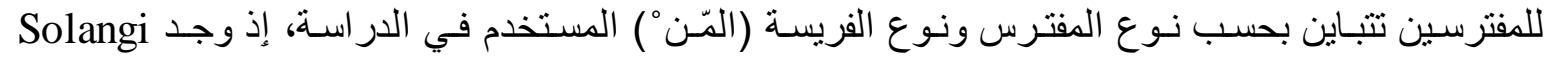

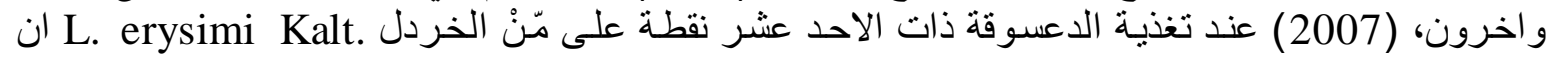

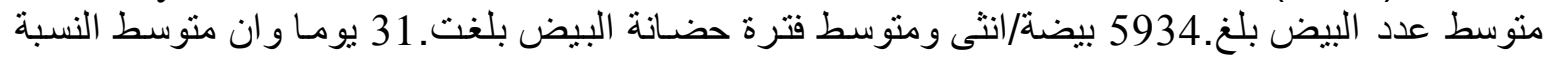

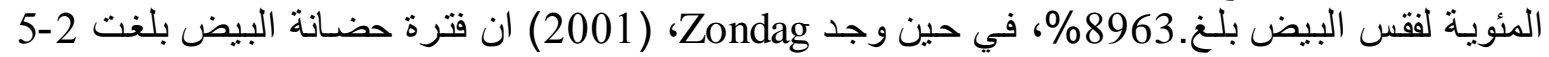

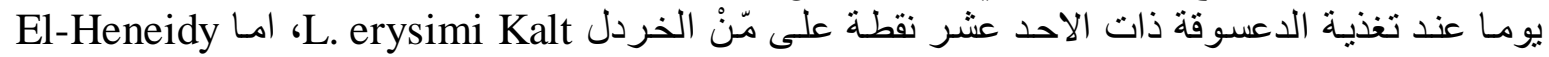

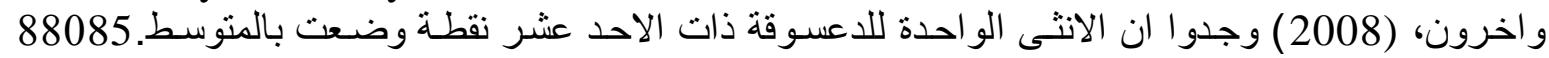

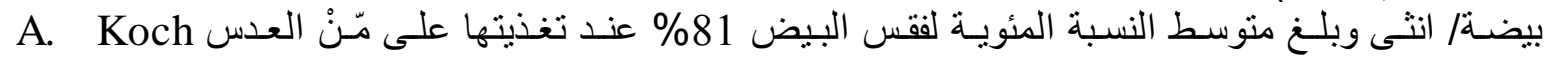

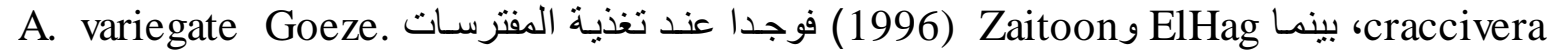

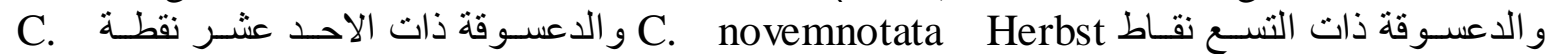

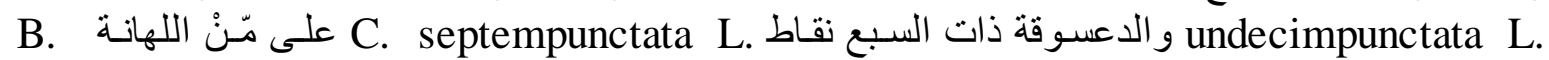

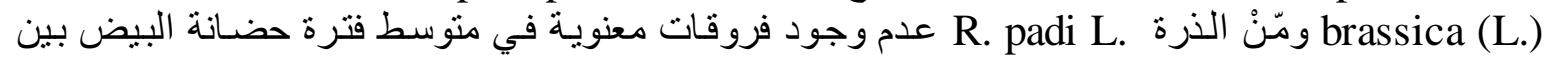

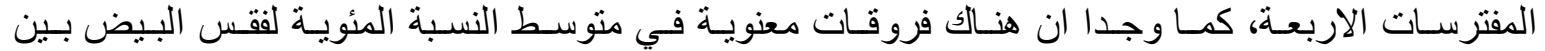

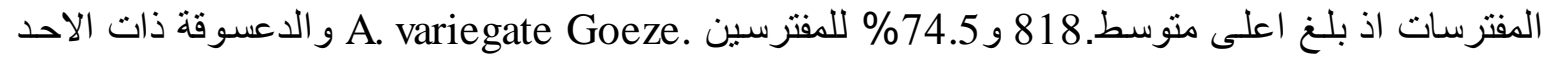

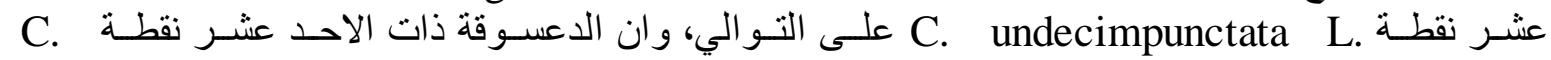
undecimpunctata L.

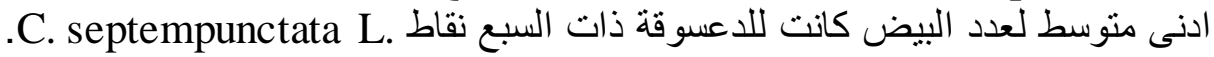




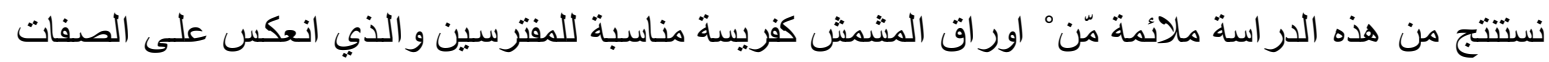

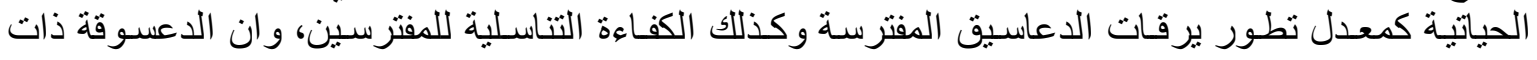

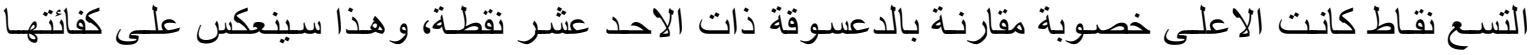

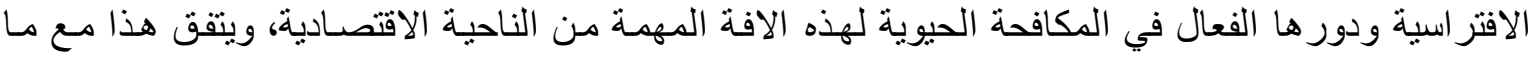

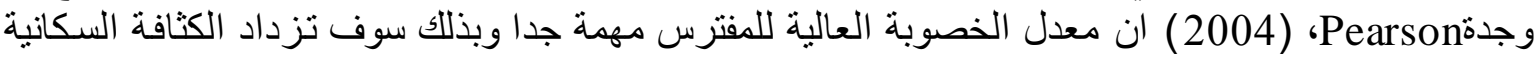
للمفترس بسر عة كبيرة عند توفر الفريسة في الحقل وتلعب دور ا فعالا في تتظيم كثافتها السكانية.

\title{
EFFECT OF FEEDING Coccinella novemnotata Herbst, AND C. undecimpunctata L. (COLEOPTERA: COCCINELLIDAE) ON Hyalopterus pruni Geoffr. (HEMIPTERA: APHIDIDAE) ON SOME BIOLOGICAL CHARACTERS OF THE TWO PREDATORS
}

Juma'a T. Mohammad

Plant Prot. Dept., College of Agric. and Forestry, Mosul Univ., Mosul, Iraq

Email:jumaa taha@yahoo.com

\begin{abstract}
Results of studying the extent suitability of Hyalopterus pruni Geoffr. (Hemiptera: Aphididae) as suitable prey to the Lady beetles Coccinella novemnotata Herbst and Coccinella undecimpunctata L. (Coleoptera:Coccinellidae) on some biological characters of two predators, showed that there is no significant effects between the means of larval development period which was 2.8,2.6,3.4 and 3.6 days for the fourth larval stages period of C. novemnotata Herbst respectively, undecimpunctata $\mathrm{L}$. in comparison with 2.6,2.8, 3.4 and 3.6 days for $\mathrm{C}$. respectively, but there is significant effects between the means of pupal period which was 4.2 and 5.2 days for two predators respectively, the results also shows a significant effects on the means of oviposition period which was 26.67 and 22.33 days for C. novemnotata Herbst and C. undecimpunctata L. respectively but there is no significant effects between the means of preoviposition and postoviposition period which were 4.67,4.33,4.33 and 5.00 days for C. novemnotata Herbst and C. undecimpunctata $\mathrm{L}$. respectively and thus the significant effects on the means of number of egg per female, dialy deposited and eggs hatching percentage which reached $2030.67 \mathrm{egg} /$ female,67.17egg/female/day and $89.22 \%$ for C. novemnotata Herbst respectively in comparison with $758.00 \mathrm{egg} / \mathrm{female}, 36.46 \mathrm{egg} / \mathrm{female} /$ day and $85.54 \%$ for C. undecimpunctata L. respectively but there is no significant effects between the means of incubation period which reached 2.35 and 2.70 days for C. novemnotata Herbst and C. undecimpunctata L. respectively.
\end{abstract}

Keywords: Coccinella novemnotata, C. undecimpunctata, Hyalopterus pruni, Biological characters.

Received: 8 /1/2014, Accepted 17/12/2017

المصادر

Anonymous (1993). SAS User's Guide: Statistics SAS Institute Inc., Cary, North Carolina, Pages 1025, USA. 
Aslan, M.M. and N.Uygun (2005). The aphidiophagus coccinellid (Coleoptera: Coccinellidae) species in Kahramanmaras, Turkey. Turkish Journal Of Zoology, pp: 1-8.

Bahy El-Din, I. A. E. (2006). Studies On The Biology And Feeding Capacity Of Some Coccinellid Species. M.Sc. Thesis, Faculty of Agriculture, Moshtohor Benha University, Egypt.

ElHag, E.T.A and A.A. Zaitoon (1996). Biological parameters for four Coccinellid species in Central Saudi Arabia. Biological Control, Vol.7 (3), pp. 316-319.

El-Heneidy, A. H.; Hafez, A. A.; F. F. Shalaby and I. A. B. El-Din (2008). Comparative biological aspects of the two coccinellid species; Coccinella undecimpunctata L. and Hippodamia convergens Guer. under laboratory conditions. Egyptian Journal of Biological Pest Control, 18 (1):51-59.

Evans, E.W., Stevenson, A.T. and D.R. Richards (1999). Essential versus alternative foods of insect predators: benefits of a mixed diet. Oecologia 121: $107-112$.

Giorgi, J. A.; Vandenberg, N. J.; McHugh, J. V.; Forrester, J. A.; Slipinski, S. A.; Miller, K. B.; Shapiro, L. R. and M. F. Whiting (2009).The evolution of food preferences in Coccinellidae. Biological Control, 51:215-231.

Heinz, K. M. and Salom, F. G.(1996). Performance of the predator Delphastus pusillus on Bemisia resistant and susceptible tomato lines. Experimentalis et Applicata Entomologia, 81: 345-352.

Kalushkov, P. and I.Hodek (2001). New essential aphid prey for Anatis ocellata and Calvia quatuordecimguttata (Coleoptera: Coccinellidae). Biocontrol Science Technology, 11: 35-39.

Liu, T. X.; Stansly, P. A.; Hoelmer, K. A. and L. S. Osborne (1997). Life history of Naphaspis oculatus (Coleoptera: Coccinellidae), a predator of Bemisia argentifolii (Homoptera: Aleyrodidae). Annals of the Entomological Society of America, 90: 776-782.

Mari, J.M.; Rizvi, N.H.; Nizmani, S.M.; Qureeshi, K.H. and M.K. Lohar (2005). Predatory efficiency of Menochilus sexmaculatus Fab. And Coccinella undecimpunctata L. (Coccinellidae: Coleoptera) on alfalfa aphid, Therioaphis trifolii (Monell.), Asian Journal of Plant Science 4(4):365-369.

Nielsen, F.H.; Hauge, M.S. and S. Toft (2002). The influence of mixed aphid diets on larval performance of Coccinella septempunctata L. (Coleoptera: Coccinellidae). Journal of Applied Entomology, 126: 194-197.

Omkar, G. and S. Srivastava (2003). Influence of six aphid prey species on development and reproduction of lady beetle, Coccinella undecimpunctata L., Biocontrol, 48:379-393.

Ozder, N. and O. Saglam. (2003). Effects of aphid prey on larval development and mortality of Coccinella undecimpunctata L. and Coccinella septempunctata L. (Coleoptera: Coccinellidae). Biocontrol Science Technology, 13:449-453.

Pearson, S.A. (2004).The Columbia Electronic Encyclopedia. 6th. Edn. Columbia University Press. Pearson Edu. Columbia, USA.

Roy, S.; Mukhopadhyay, A.; Das, S. and G. Gurusubramanian (2010). Bioefficacy of coccinellid predators on major tea pests. Journal of Biopesticides, 3: 3336. 


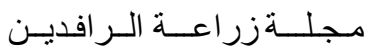
المجلد (46) العدد (3) 2018

Slipinski, A. (2007). Australian ladybird beetles (Coleoptera: Coccinellidae): their biology and classification. Australian Biological Resources Study, Canberra. 286 pp.

Solangi, B. K.; Ghani L. A.; M. K. Lohar (2007). Biology of 11-Spotted Beetle Coccinella undecimpunctata L. (Coccinellidae: Coleoptera) on Mustard Aphid Lipaphis erysimi Kalt. Journal of Applied Science, 7(20):3086-3090.

Zondag, R. (2001). Eulachnus brevipilosus Berner (Hemiptera: Aphididae). Pine aphid. New Zealand Forest Service, Forest and Timber Insects in New Zealand No. 55. 